\title{
Amartya Kumar Sen, Premio Nobel de Economía 1998
}

\begin{abstract}
Álvaro Magaña
"Si queremos utilizar el equilibrio de mercado, competitivo para conseguir cualquier óptimo social, tenemos que disponer de la distribución inicial de recursos correcta y, dependiendo de cómo y cuánto de equitativos sean nuestros objetivos, tal cosa podría exigir una reasignación total de las relaciones que hayamos heredado históricamente". Bienestar, justicia y mercado.

Amartya K. Sen
\end{abstract}

\section{Resumen}

En este artículo, el autor analiza los trabajos y planteamientos del profesor Amartya K. Sen, que el pasado octubre recibió el Premio Nobel de Economía de 1998. En sus estudios, el profesor Sen ha cuestionado de manera clara y categórica los métodos y principios fundamentales característicos del análisis económico neoclásico tradicional. Más aún, en la orientación de sus trabajos e investigaciones se evidencian sus serias dudas sobre las bondades de las corrientes prevalecientes, identificadas con el llamado neoliberalismo y concretadas, en la práctica, por el dogma estrictamente cumplido de una obediencia ciega a las fuerzas del mercado.

1. Ex profesor de Economía y de Ciencia de la Hacienda de la Facultad de Jurisprudencia y Ciencias Sociales de la Universidad de El Salvador, 1956-1960. Director Adjunto del Departamento de Asuntos Económicos de la Unión Panamericana (Organización de Estados Americanos, OEA) y Director Ejecutivo del Programa Conjunto de Tributación OEA-BID-CEPAL, 1961-1965. Presidente del Banco Hipotecario de El Salvador, 1965-1982. Director del Banco Central de Reserva de El Salvador, 1976-1982. Presidente de la República de El Salvador, 1982-1984. 
El premio que sin duda es el más prestigioso que se concede anualmente a quienes han hecho contribuciones importantes para la paz, la literatura y en distintas ramas de las ciencias, es el que lleva el nombre de quien lo estableció a principios del siglo: Alfred Nobel, aunque el premio de Economía se estableció hasta 1969.

Motivaciones de carácter personal nos dieron el pretexto, en 1995, para escribir un trabajo sobre el Premio Nobel en Economía, de ese año; pero, en verdad, ese trabajo fue nuestro primer intento de referimos, por escrito, criticamente a las políticas neoliberales que se han entronizado en nuestro país curiosamente con la paz, que terminó un conflicto que según nos han dicho nadie ganó.

En esa oportunidad planteábamos algunos aspectos de esos esquemas mal llamados de modemización, patrocinados por el binomio de los organismos financieros, de todos conocidos, que logran sus cuestionables propósitos, premiando a los obedientes y "bien portados" con financiamientos. En ese trabajo nos referimos al fin de la redistribución con la reforma tributaria neoliberal; a la reducción acelerada y atolondrada de los impuestos de importación, que no fue más que una calendarización apresurada por la obediencia ciega y servil de quienes, entre nosotros, tomaron las decisiones equivocadas y peor calendarizadas, desde nuestro punto de vista, para la globalización, cuando provocaron un aumento en el consumo. Esto incidió en forma significativa sobre nuestra capacidad de ahorro y, lo que es grave también, resultó en un considerable déficit comercial, afortunadamente aliviado por las remesas de quienes hoy llamamos "hermanos lejanos".

Además, y probablemente lo peor, nuestras pequeñas y medianas empresas quedaron expuestas a una competencia desventajosa por quienes podían producir a menores costos que nosotros, cuando las empresas salvadoreñas, además de su limitado desarrollo tecnológico, deben pagar los intereses elevados que los personeros de nuestra Banca Central debían mantener a esos niveles, para restringir la demanda crediticia y, de este modo, cumplir lo que se han obligado a acatar dentro de las metas que les fijan para la inflación, el tipo de cambio, etc.; por tanto, como dijimos en otra oportunidad, resultaron más "fondistas" que el Fondo.

Después de ese trabajo desarrollado en 1995, en los últimos años hemos reiterado en otros nuestra preocupación por las consecuencias peligrosas que conllevan esas políticas, como lo demuestra la dura experiencia de tantos países, comenzando con México, en 1995, seguido por todos los del 97 en Asia, incluso los tres tigres, para llegar después, en el pasado agosto, a Rusia, sin olvidar a Brasil, el cual también confrontó problemas en 1999. Afortunadamente debemos reconocer que los temores de una depresión, que ensombrecieron la reunión anual del Fondo y del Banco Mundial en octubre pasado, al menos como ocurrió en los casos citados, no creo que sean motivo de preocupación para nosotros, pues los personeros salvadoreños responsables de nuestra política económica en los últimos años - no sabemos si a propósilo, por casualidad o equivocación- han sido muy exitosos y extremadamente eficientes para lograr que la inversión extranjera haya alcanzado niveles mínimos. Así, en lo que respecta a nosotros, y gracias a nuestra buena suerte, no debemos preocupamos por el riesgo de un reliro de esa inversión con todas sus consecuencias, que hemos observado en muchos países, porque no nos podrá ocurrir.

Deberíamos tener claro que la globalización es un hecho, pero el neoliberalismo es sólo una forma de encarar la globalización. No es la verdad revelada, como parece o quieren creer muchos, como los personeros del Banco Central, quienes, encerrados en su mercadolatría dogmática, han pasado repitiendo hasta el cansancio los datos envidiables de los niveles de inflación y de la estabilidad del tipo de cambio, ignorando aquellos que interesan a la generalidad que siente los efectos negativos de las políticas neoliberales: altas tasas de interés, importaciones crecientes, exportaciones estálicas, inversiones extranjeras inexistentes, etc. y, por último, el resultado final: un incremento sostenido y significativo de la pobreza que ahora, después que el "Mitch" la ha puesto en evidencia, pareciera que será objeto de atención ${ }^{2}$.

2. En nuestro estudio, publicado en 1988, "Los límites constitucionales de la autonomía del Banco Central", reproducimos un cuadro que contiene datos estadísticos muy reveladores sobre la mínima inversión extranjera directa en nuestro país, en los últimos años. Respecto a las exportaciones, lo único que aumentó fue la maquila, además de sus efectos negativos, como los bajos salarios, etc.; por otro lado, hay que recordar que se trata de inversión "golondrina". 
En varios trabajos anteriores, nuestros planteamientos han sido realmente cuestionamientos que estábamos obligados a hacer, cuando en los últimos años están a la vista los resultados y las consecuencias de la libertad irrestricta del mercado, pues nosotros nunca hemos aceptado ni creído que éste pueda resolver los problemas de una sociedad, al menos si no queremos ignorar que nuestro crecimiento y desarrollo debería ser en términos de una justicia igualitaria muy deseable.

En este artículo nos referiremos específicamente a planteamientos y análisis de los problemas económicos que consideramos no son muy conocidos, pero que son de mucha actualidad por las razones que puntualizaremos después. Queremos referimos a los aspectos más relevantes del pensamiento del profesor Amartya K. Sen, que el pasado octubre recibió el Premio Nobel en Economía de 1998, convirtiéndose así en el laureado No. 43 en esa diciplina.

Al conocer algunos de los muchos libros y artículos publicados por el profesor Sen, no quedan dudas de que se trata de un merecido reconocimiento a sus valiosos aportes a las ciencias económicas, durante su larga docencia académica en prestigiosos centros de educación superior, como la London School of Economics y las universidades de Oxford y Harvard. En este artículo queremos hacer solamente una relación resumida de uno de los aspectos estudiados por el profesor hindú, entre su amplia producción bibliográfica, cuando en el estudio introductorio de uno de sus libros se hace el significativo señalamiento respecto a la obra del profesor Sen, "que puede ser calificada, sin temor a exagerar, de monumental".

Nosotros nos limitaremos a mencionar los trabajos que consideramos más representativos y que están relacionados con aquellas áreas que caracterizan su línea original de pensamiento y a los que seguramente deben atribuirse fundamentalmente la concesión del prestigioso premio. Lo anterior no es una tarea fácil, cuando ha incursionado en la teoría de la elección social, la economia del bienestar, los problemas de los alimentos, las hambrunas, el hambre y la pobreza, el crecimiento y el capital; la economía de la familia y las divisiones sociales; la evaluación de proyectos y el análisis coste-beneficio; la educación y la planificación de la mano de obra y el empleo; la

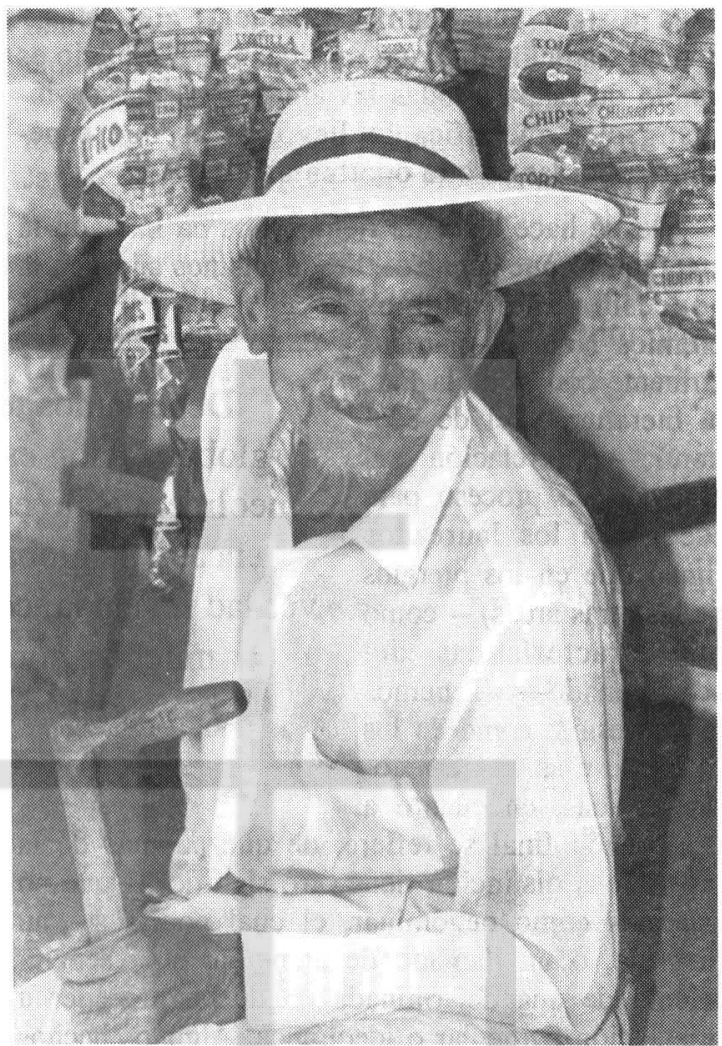

ética y la filosofía moral, la filosofía legal, política y social, entre otros.

Pero lo que es verdaderamente significativo ha sido señalado, cuando en el estudio introductorio citado se indica: "Puede decirse que Sen ha cambiado, posiblemente para siempre, algunas ramas de la Economía como, por ejemplo, la Economía del bienestar, la concepción y explicación de las hambrunas y la interrelación entre la Ética y la Economía". Debe reconocerse la amplia variedad de materias de interés que aborda el profesor Sen, lo cual hace difícil sintetizar, dentro de los límites de un artículo, sus valiosas contribuciones, algunas de mayor interés para el filósofo, otras para el economista o el estudioso de la economía política. Por eso nos referiremos, en forma sumaria, a un aspecto de sus planteamientos del cual debe señalarse su impacto, que seguramente ha sido mayor, aun cuando se trate de ignorar el que se 
haya distanciado significativamente del pensamiento tradicional, y aún más cuando ese juicio es válido quizá para todas las áreas a las que su investigación científica lo llevó, áreas que esperamos abordar en otra oportunidad ${ }^{3}$.

Desde hace bastante tiempo nos ha llamado la atención lo que acontece, desde algunos años, en la concesión de algunos premios Nobel $\longrightarrow$ particularmente con el de la Paz, la literatura y el de economía- en relación con el laborioso proceso para escoger a los laureados (igual que en los premios de las otras áreas) -como las características del seleccionado-. Tenemos la impresión, como en los casos de esas tres categorías citadas, en cuanto a la decisión final se refiere, de que además de la prestigiosa distinción tiene otro significado que no sabemos cómo denominar, el cual podría ser un mensaje, o un llamado de atención, o el señalamiento de una determinada conducta, proceder u orientación particular o ideológica. Algunos hechos nos hacen pensar de esta forma, como el haber otorgado el premio a Rigoberta Menchú, el no dárselo a Borges o el concedérselo a Saramago, en 1998.

Mencionamos lo anterior porque, como precisaremos después, el pensamiento nada ortodoxo del profesor Sen es objeto de la que seguramente es la máxima distinción científica, justamente cuando el neoliberalismo - con su infalible mer- cado- comienza a formar parte de otro capítulo más de la historia del pensamiento económico, igual que ocurre siempre cuando las utopías terminan, aunque esta vez no era utopía ${ }^{4}$.

Es interesante que Jeffrey Sachs, Director del Harvard Institute for International Development, en su artículo "Las causas reales del hambre"

("The real causes of famine", Time, 26 de octubre de 1998, p. 29), hizo el significativo señalamiento al referirse al Premio Nobel de Sen: "En un mundo en el cual 1.5 billones ( 1500 millones) de gente, subsiste con menos de \$1 (un dólar) al día, este premio Nobel no puede ser sólo una celebración para un erudito admirable sino también una llamada para atender las urgentes necesidades y esperanzas de los pobres del mundo" (traducción hecha por el autor).

\section{EJames Galbraith, hijo de John Kenneth Gal-} braith, probablemente el más conocido institucionalista postkeynesiano, en su reciente libro $\mathrm{Cre}$ ated Unequal, en el cual estudia cómo el nivel elevado de desempleo ha producido desigualdad en los ingresos, comienza el último capítulo del libro con el planteamiento siguiente, que mueve a reflexión: "La Economía, como disciplina y como profesión tiene una larga tradición de conformidad en el sistema social prevaleciente, que nos ha mantenido únicamente en el caso raro de alguna rebelión. Ahora necesitamos una rebelión"s .

3. El estudio introductorio hecho al libro del profesor Amartya Kumar Sen, Nueva Economía del Bienestar (Universidad de Valencia, 1995, p. 11), es de José Casas Pardo. En la introducción a otro libro del profesor Sen, Bienestar, justicia y mercado (Ediciones Paidós ICE, de la Universidad Autónoma de Barcelona), Damián Salcedo expresó, en 1997, que era el "pensamiento verdaderamente original de un autor que creo va camino de convertirse en un clásico de la filosofía política de nuestro siglo” (p. 13). Este libro consta de dos conferencias de Sen, la de John Dewey, en 1984, en la Universidad de Columbia; y la Conferencia John Hicks, en Oxford, en 1990; mientras que el artículo titulado "Justicia: medios contra libertades", forma parte de otro más extenso: "The territory of Justice".

4. Después de redactar el anterior planteamiento en el último número de Challenge, leímos un artículo muy detallado titulado "Who wins the Nobel Prize?" (pp. 23-40), cuyo autor es el economista belga Johan Van Gompel. En el mismo número de Challenge aparece un interesante planteamiento de Bárbara Bergmann, en su artículo "Abolish the Nobel Prize for Economics" (pp. 52-57).

5. James Galbraith, "Created unequal: The crisis in American Pay", New York: Free Press, 1998, p. 263 (traducción hecha por el autor). 
En este orden de ideas, desde el inicio queremos destacar el claro y categórico cuestionamiento que el profesor Sen hace en sus estudios sobre los métodos y principios fundamentales característicos del análisis económico neoclásico tradicional prevaleciente, aceptado en forma general en la actualidad. Más aún, en la orientación de sus trabajos e investigaciones se evidencian, sin duda alguna, sus serias dudas sobre las bondades de las corrientes prevalecientes, identificadas con el llamado neoliberalismo y concretadas, en la práctica, por el dogma estrictamente cumplido de una obediencia ciega a las fuerzas del mercado; no obstante que eso produzca todas las consecuencias negativas que estamos viendo. Es difícil explicarse que después de lo ocurrido en tres continentes, en 1997 y 1998, aún no exista un concenso generalizado sobre la orientación que deberían tener las políticas económicas, a fin de que se pueda evitar la continuación y propagación de las crisis económicas ocurridas en muchos países. Quizás el desconcierto es peor que cuando se derrumbó aquel muro, en noviembre de 1979.

Nuestro interés principal radica en las observaciones del profesor Sen sobre la eficiencia del mercado que, juntamente con los estudios sobre la pobreza, las hambrunas y la desigualdad, constituyen los campos en los cuales probablemente el profesor hizo los señalamientos fundamentales más significativos. Con base en lo anterior, francamente no sabemos qué pensar sobre la curiosa coincidencia, que seguramente no pasó desapercibida para muchos, de que cuando se otorga el premio a un economista, con la línea del pensamiento del profesor Sen, al mismo tiempo en la reunión anual del Banco Mundial y del Fondo Monetario Intemacional - realizada en la primer semana del mes de octubre- están tratando inútilmente de encontrar soluciones a las situaciones provocadas por el binomio mencionado, mediante las políticas del neoliberalismo y libre mercado, en Malasia, Indonesia, Corea del Sur, Japón, México, Brasil, etc. Además, tampoco podemos ignorar la curiosa coincidencia de que la izquierda domina sin ninguna duda en Europa, y que sólo España e Irlanda tienen gobiernos derechistas de los quince países que conforman la Unión Europea ${ }^{6}$.

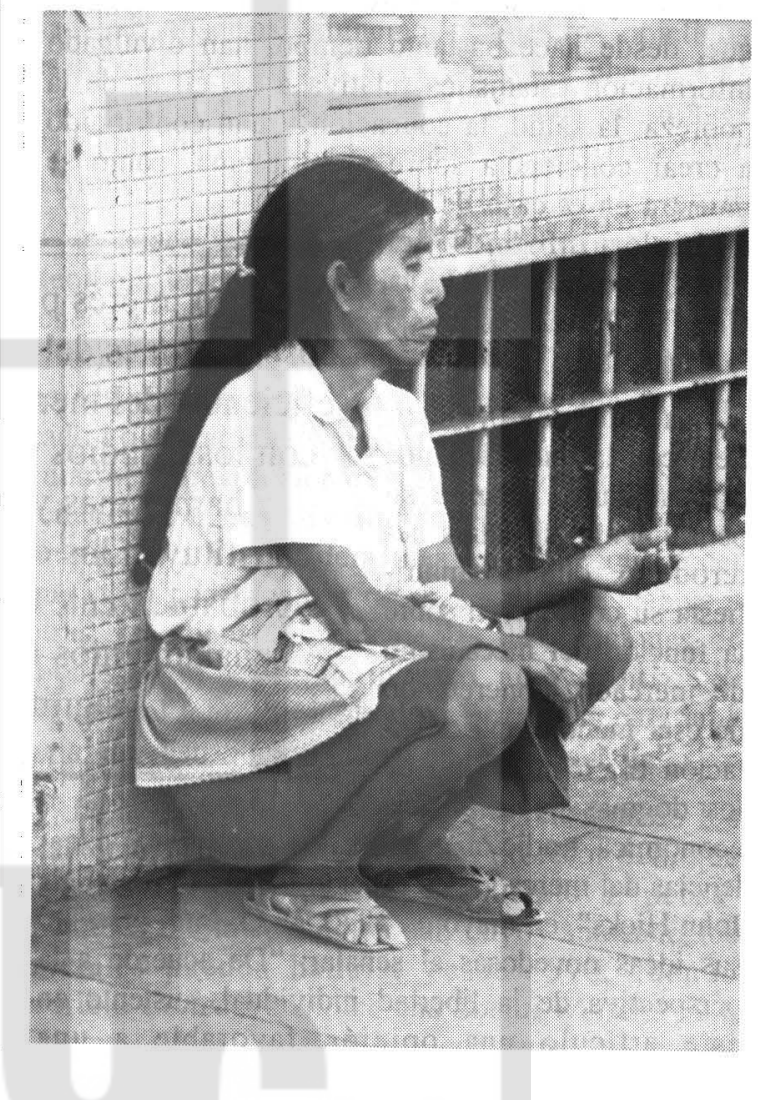

6. Son interesantes los planteamientos de Stephen Herzenberg, John Alic y Howard Wial, publicados en su reciente libro Nuevas reglas para una nueva economía (New rules for a New Economy: Employment and Opportunity in Postindustrial America. Ithaca: Cornell University/ILR Press, 1988). En esa obra, los autores sostienen que la educación y los mercados libres no son suficientes y proponen una serie de reformas institucionales. En un reciente arlículo titulado "Un nuevo trato para una nueva economía" ("A new deal for a new economy", Challenge, marzo-abril de 1999, pp. 102-127) comentan lo siguiente de su libro: "Nuestro Nuevo Trato para una Nueva Economía comparte algunas características con la del mismo nombre de Rossevelt: una preocupación por combinar equidad y seguridad con eficiencia y crecimiento y, la creencia de que el gobierno puede ayudar a resolver los problemas económicos" (p. 128) (traducción hecha por el autor), y concluyen su artículo afirmando que "Ha llegado el tiempo para crear un Nuevo Trato para la Nueva Economía". Jeffrey Madrich, editor de Challenge, al comentar los puntos de vista de los autores del libro citado dice que "Los comentarios incisivos del trío respecto a lo que hay que hacer pueden ofender la tendencia prevaleciente. Pero, entonces, la tendencia prevaleciente establecida ha estado equivocada por un largo tiempo" (p. 5) (traducción hecha por el autor). 
Por otra parte, es significativo que, aun cuando es desde hace relativamente poco tiempo, las propias entidades crediticias internacionales, como el Fondo Monetario Internacional (FMI), el Banco Mundial (BM) y el Banco Interamericano de Desarrollo (BID) -que han propiciado y obligado la adopción de las políticas neoliberales a cambio del financiamiento a nuestros países-, han comenzado a mostrar alguna atención, a su modo, respecto al problema de la pobreza puesto que ya se atreven a mencionarlo. Distinta es la actitud de los organismos de Naciones Unidas que, desde hace bastante tiempo, han divulgado información estadística relativa a las cifras de la pobreza, la salud, la educación y han contribuido a crear conciencia sobre el problema, como se muestra en el caso de los múltiples informes del PNUD, de la CEPAL, la UNCTAD, la UNESCO, la OMS, la OIT, etc.

La cita del profesor Sen, con la que iniciamos este artículo, resume su pensamiento, sin duda heterodoxo, cuando manifiesta su concepción sobre la función del mecanismo de mercado, concretando de esta manera una orientación diferente a uno de los dogmas de la ciencia económica tradicional sobre las virtudes y excelencias del mercado. En el texto de su "Conferencia John Hicks", en mayo de 1970, en Oxford, presentó sus ideas novedosas al señalar: "De acuerdo a la perspectiva de la libertad individual, sustento en este artículo una opinión favorable a una reinterpretación de lo que se supone realiza el mecanismo de los mercados competitivos y también en favor de que se revalúe lo que podríamos esperar de ellos, que puedan lograr que se realice"7 (traducción hecha por el autor).

El profesor Sen señala que: "A menudo se utiliza de un modo enérgico el lenguaje y la retórica de la libertad para defender el mecanismo de mercado..." Pero agrega que la teoría económica de la asignación de recursos ha tendido a vincularse sólidamente a una estructura normativa "bienestarista". Y precisa a continuación: "Los éxitos y fracasos de los mercados competitivos, se juzgan enteramente por logros de bienestar individual (por ejemplo en términos de la optimalidad de Pareto medida en utilidades) y no por lo que hacen para fomentar la libertad individual".

Para relacionar en forma resumida los puntos de vista del profesor Sen, debemos comenzar por identificar la base en la que los economistas se apoyan para defender las excelencias que le atribuyen al mercado, lo cual el Nobel 1998 resume así: "La evaluación de los fundamentos del mecanismo del mercado en la economía moderna, se basa en gran medida en el llamado 'teorema' fundamental de la economía del bienestar", pero debemos señalar que "este teorema trata sólo de mercados completamente competitivos y se centra en lo que sucede cuando los mercado están en equilibrio y no cuando están desequilibrados". El profesor señala que el teorema tiene dos partes; la primera proposición la denomina "teorema directo", que consiste en que bajo ciertas condiciones especificadas (ausencia de externalidades, es decir ausencia de interdependencias que no sean

7. Amartya K. Sen, Bienestar, justicia y mercado, Ediciones Paidós, ICE de la Universidad Autónoma de Barcelona, 1997. p. 123 y ss. La versión revisada de la conferencia fue publicada en Oxford Economics Papers 45 , 1993, pp. 519-541, con el título "Markets and freedoms: achievements and limitations of the market mechanism in promoting individual freedorns" ("Mercados y libertades. Logros y limitaciones del mecanismo de mercado en el fomento de las libertades individuales", incluido en Bienestar, justicia y mercado, p. 123 y ss.) La versión original en idioma inglés se incluye (en ese idioma), en Amartya Kumar Sen, Nueva Economía del Bienestar, Escritos seleccionados, Universidad de Valencia, 1995, p. 247 y ss. En la sección 2 de ese trabajo, titulado "Markets and Welfarist efficiency" ["Mercado y eficiencia de bienestar"], es donde el profesor Sen desarrolla la cuestión específica de nuestro especial interés en el presente artículo. 
de mercado), todo equilibrio competitivo de mercado es "eficiente según Pareto" (también llamado "óptimo de Pareto"). Para Pareto, señala Sen, un estado o situación (state of affairs) se considera o define como eficiente, "si es el caso de que, comparado con él, no se puede aumentar la utilidad de nadie, sin reducir la utilidad de los demás". O dicho en otra forma, el "teorema directo" dice que "bajo las condiciones especificadas, no es posible realizar, mejoras generales sin conflicto [juzgadas en términos de utilidades individuales], a partir de un equilibrio de mercado competitivo cualquiera"8

El profesor advierte que la segunda parte del teorema es más compleja cuando refiere que dadas algunas condiciones (que incluyen la ausencia de externalidades, pero también la ausencia de economías de escala significativas), todo resultado eficiente, según Pareto, es un equilibrio competitivo para algún conjunto de precios y respecto a una distribución inicial de recursos, y agrega: “Este 'teorema inverso' ha sido considerado típicamente como la tesis más importante en favor del mecanismo de mercado". Es decir, no importa cual o que estado eficiente, según Pareto, especifiquemos, pues es posible un equilibrio de mercado competitivo que produzca precisamente ese estado, eligiendo adecuadamente una distribución inicial de recursos. Y agrega el profesor: Este "teorema inverso" ha sido considerado típicamente como el argumento más importante en favor del mecanismo de mercado.

En el párrafo siguiente se resume lo más importante, novedoso y esencial de los planteamientos del profesor Sen cuando enjuicia críticamente los criterios tradicionales del mecanismo de mercado así: "El 'teorema directo' —en cuanto a que todo equilibrio de mercado competitivo es eficiente según Pareto- quizás resulte no ser un trofeo o triunfo formidable para el mecanismo de mercado, cuando, o puesto que, es difícil considerar que la eficiencia paretiana sea suficiente para la optimabilidad social. La eficiencia pa- retiana es totalmente desinteresada (o indiferente) en la distribución de utilidades (o de rentas o de cualquier otra cosa) y tiene un desinterés total en la equidad"9.

Las palabras transcritas al inicio de este artículo plantean una apreciación de fondo del problema, cuando se hace el importante señalamiento de que "si queremos utilizar el equilibrio de mercado competitivo para conseguir óptimo social, tenemos que disponer de la distribución inicial de recursos correcta". Ahora bien, el grado de equidad que nosotros asignemos a nuestros objetivos es lo que determinará si es necesaria una reasignación total del sistema de relaciones de propiedad, que modifique el sistema de relaciones que hayamos heredado históricamente.

A continuación Sen precisa puntualmente: "El 'teorema inverso', pues [o en consecuencia], pertenece a un 'manual' de revolucionario". Y agrega: "No me preocupa el hecho sociológico de que los defensores más entusiastas del mecanismo de mercado no se caractericen precisamente por ser particularmente revolucionarios [típicos] que demandan [cuando exigen] distribuciones radicales de la propiedad". Y considera más relevante reconocer que si no podemos por razones políticas, legales u otras, "reordenar libremente las distribuciones de recursos, el teorema inverso no garantiza ni siquiera el logro limitado de la eficiencia paretiana para una distribución inicial de recursos dada".

En los párrafos previos hemos relacionado los planteamientos significativos del profesor Sen, que constituyen un serio cuestionamiento al pensamiento económico tradicional, cuyos antecedentes iniciales pueden hallarse desde 1970 , cuando escribió Collective choice and Social Welfare (San Francisco: Holden Day), que constituyó un avance significativo en el estudio de la teoría de la elección social, que se había iniciado con las investigaciones de K.E. Arrow, en 1951, en su clásico de aquellos días, Social choice and individual values ${ }^{10}$.

8. Amartya K. Sen, Bienestar, justicia y mercado, p. 126.

9. Las expresiones entre paréntesis son traducciones alternativas del original en inglés, hechas por el autor, ya que considera que son más claras que las que se encuentran en castellano en Bienestar, justicia y mercado, p. 126. Otras veces se transcribe el texto de la expresión en inglés, del texto original, cuando la traducción no capta fielmente el sentido de una expresión.

10. En 1976, Alianza (Madrid) publica en castellano Elección colectiva y bienestar social, que es el libro citado de Sen, publicado en 1970. La versión inglesa de la obra de Arrow fue publicada por Wiley: New York, en castellano, con el título Elección social y valores individuales, a cargo del Instituto de Estudios Fiscales (IEF) 
En el mismo año 1970, Sen publica también "The Impossibility of a Parentian Liberal"11, que como ha sido calificado "pronto se revelaría como la conceptualización de un problema que afecta de una manera profunda a los supuestos básicos de la ciencia económica". Esto es así al mostrar que una de esas convicciones incuestionables para los economistas es problemática, como es el caso de la creencia de que eficiencia y libertad van de la mano en las decisiones sociales. Sus reflexiones sobre las dificultades para reconciliar ambos principios lo llevan a conclusiones contrarias al modo habitual de como la ciencia económica considera el comportamiento humano, que se convierten en el inicio de su pensamiento filosófico. Un buen ejemplo es "Rational Fools: A critique of the Behavioural Foundations of Economic Theory" en Philosophy and Public Affairs (6, 1977, pp. 317-344) ${ }^{12}$.

Aun cuando se considere repetitivo, es muy útil referirnos aún a la relación del profesor español, Salcedo Megales, con los aspectos más relevantes de los originales aportes del profesor Sen en lo que se refiere al estudio de los mercados competitivos y la medida en que la libertad y la eficiencia son realizados, sin olvidar, para relacionarlos, lo esencial de la argumentación de los economistas, que defienden las excelencias del mercado cuyos planteamientos, o sea la doctrina "oficial", descansa en los teoremas básicos siguientes de la economía del bienestar, a los que nos referimos a continuación siguiendo la relación del profesor español sobre los teoremas mencionados: Primer teorema Básico: Cada equilibrio competitivo es un óptimo de Pareto. Segundo teorema Básico: Cada óptimo de Pareto es un equilibrio competitivo.

Ahora bien, respecto a lo anterior podemos decir con el profesor Sen que, si un determinado estado social es un óptimo de Pareto, lo único que se asegura es su eficiencia, es decir que no hay otro estado social en el que todos estén mejor 0 , dicho de otra forma, nadie puede mejorar sin que otro empeore.

Al primer teorema se le ha criticado su carácter abstracto, que no concuerda con la realidad de los hechos; las preferencias de los consumidores no están dadas, más bien han sido creadas; tampoco la economía es estática cuando las tecnologías y preferencias cambian. Por otra parte, como bien se ha señalado, el supuesto de la competencia es muy cuestionable, pues es bastante irreal por el hecho de que la economía real está llena de monopolistas $\mathrm{y}$, de igual modo, la conducta, tanto de los consumidores como de las empresas, depende de la existencia de extemalidades, o sea que va más allá de sus relaciones de mercado.

Pero la segunda clase de críticas es indudablemente más importante, pues cuestionan el valor moral del mercado. Estas críticas, respecto al valor moral del mercado, señalan la ignorancia o indiferencia de que son objeto los aspectos distributivos en el teorema mencionado. De esta manera se ha señalado que "un equilibrio de mercado puede que sea un óptimo de Pareto, pero el criterio paretiano no dice nada sobre la justicia de ese estado social". Y, además, que "Hay muchos equilibrios competitivos que, ciertamente, son óptimos de Pareto, dependiendo de la distribución inicial de recursos. Pero unos son más justos que otros".

En seguida se plantea una interrogante, la cual dejamos que el lector califique: "¿Qué dice el primer teorema sobre los planes de producción que atienden con cuidado los deseos de los ricos de productos lujosos, mientras que apenas atienden las necesidades de los pobres de alimento, vivienda o atención sanitaria?". Como claramente es un tipo de pregunta que, en principio, está más allá de las

Madrid, 1974. Kaushic Basu en su arlículo "Amartya Sen, Economics Nobel laureate 1998" (Chanllenge, marzo-abril 1999, pp. 41-51) se refiere a la obra del profesor Scn, Collective Choice and Social Welfare, como su clásico libro, su más importante libro que califica así: "Es un trabajo de inmensa elegancia, combinando la lógica formal, la economía del biennestar y la filosofía moral" (p. 42) (traducción hecha por el autor).

11. En el Journal of Political Economy, de la Universidad de Chicago, (78, 1970, pp. 152-157). Reproducido en Nueva Economía del Bienestar, pp. 131-136. La versión castellana: "La imposibilidad de un liberal paretiano" está incluida en F. Hahn Y M. Hollis (editores), Filosofía y Teoría Económica, México: Fondo de Cultura Económica, 1986.

12. Publicada su traducción como "Los tontos racionales: una crítica de los fundamentos conductistas de la teoría económica", en F. Hahn y M. Hollis, Filosofía y Teoría Económica. 
posibilidades de respuesta del teorema, se dice: "Por eso entre los economistas hallamos una tendencia a desentenderse del problema"13.

Queremos destacar que a continuación del juicio anterior se hace la observación siguiente: "Pero entonces, si lo que prentenden decir es que no hay nada problemático en la cuestión de la distribución, tendrán que añadir a los supuestos del teorema un supuesto moral más que afime que otros valores - por ejemplo, valores igualitariosno tienen importancia para el enjuiciamiento de los estados sociales".

Pero volvamos al Segundo Teorema Básico de la economía de bienestar, cuando señala que se puede alcanzar cada óptimo de Pareto por medio de un equilibrio de mercado dada una distribución inicial de recursos. Es decir, que cualquier estado social que consideremos bueno tendrá que ser, como mínimo, óptimo de Pareto. De tal manera que, en consecuencia, no importa cuál de los estados óptimos de Pareto elijamos como el más justo, pues ese estado se puede alcanzar con el mecanismo de mercado. De ahí resulta que para afrontar el problema de la insensibilidad de la justicia del mecanismo de mercado, se haya optado por señalar que un sistema inicial de transferencias de renta, junto con un sistema tributario adecuado, permitiría que el mecanismo de mercado haga su operación, puesto que el equilibrio final será siempre un óptimo de Pareto.

Entonces, el Segundo Teorema quedaría modificado y tendríamos entonces un Segundo Teorema Básico (STB) modificado así: Se puede conseguir un óptimo de Pareto justo con un equilibrio competitivo, dado un sistema adecuado de impuestos y transferencia de renta. Es decir, si determinado equilibrio de mercado es impuesto desde el punto de vista distributivo, existirá otro equi- librio de mercado, también óptimo de Pareto, más justo que aquel, y lo único que habría que hacer es modificar la distribución inical de las rentas y entonces la sola operación del mercado nos permite alcanzarlo, o dicho en otras palabras: si un determinado equilibrio de mercado u otro es injusto desde el punto de vista distributivo, al modificar la distribución inicial de las rentas podemos establecer otro equilibrio de mercado, también óptimo de Pareto, más justo, que alcanzamos por medio de la operación de mercado únicamente.

La cuestión importante es que se disiente del planteamiento anterior cuando, en su esencia, su cuestionamiento consiste fundamentalmente en considerar inaceptable la presunción de que el mecanismo de mercado es todo lo que necesitamos para alcanzar el bien común. Dicho de una manera más clara, no necesitamos de un mecanismo político.

Pues bien, si no hubiera posibilidad - por lo que sea- de modificar las redistribuciones iniciales de propiedad y renta, nos quedaría únicamente el primer teorema que vendría a sancionar lo real como racional, excluyendo cualquier motivación para introducir reformas políticas. Como señalamos antes, el Primer Teorema es claramente insuficiente desde el punto de vista distributivo, por lo que nos estaríamos engañando si lo consideramos como la prueba de la única institución social que necesitamos en el mercado.

En suma, si reconocemos, desde el punto de vista redistributivo, que la sociedad requiere de algo más que la existencia del mercado, entonces es forzoso aceptar que el valor de esa institución no se puede determinar en forma aislada, pues estaría relacionado con el valor del resto de la estructura social. Siendo así - señala el profesor Sen- debemos enfrentamos directamente con el problema de la igualdad en vez de evitarlo, ya sea

13. Las referencias de este párrafo y las siguientes son de la introducción del profesor de la Universidad de Granada, Damián Salcedo Megales, a Bienestar, justicia y mercado, titulada "La evaluación de las instituciones sociales según Amartya Kumar Sen", estudio que nos ha sido muy útil en la preparación de nuestro artículo (las cursivas son del autor). 
silenciándolo o encubriéndolo bajo algún concepto de eficiencia social.

Entre las significativas y numerosas contribuciones del profesor Sen a la ciencia económica, a propósito nos hemos limitado a relacionar únicamente sus consideraciones respecto a la ahora tan cacareada libertad de mercado, esto es porque la extensión de un artículo no lo permite y, además, porque con ello nos estamos refiriendo a una de las cuestiones de mayor actualidad.

Mientras la generalidad de ios economistas profesionales ha evaluado el mecanismo de mercado en términos de bienestar, el profesor Sen lo evalúa en términos de libertad. Esto significa que lo sitúa o, más bien, devuelve el problema al campo de la filosofía política, donde su estudio consiste en el debate de los argumentos en favor del mercado en el ámbito de la libertad. La dife-
“Qué dice el primer teorema sobre los planes de producción que atienden con cuidado los deseos de los ricos de productos lujosos, mientras que apenas atienden las necesidades de los pobres de alimento, vivienda o atención sanitaria?". nen, con otros no podemos hacerlo, pues aun cuando la operación del mercado pueda tener buenos o malos resultados, siempre serán justos sino se han violado los derechos antecedentes de las partes. Así las cosas, Salcedo señala "que este razonamiento procedimental tiene una invulnerabilidad a los argumentos empíricos que no tienen los más habituales argumentos consecuencialistas".

La crítica del profesor Sen se dirige precisamente a ese modo de razonar desde el punto de vista ético, porque ninguna institución social podría considerarse justa simplemente por la aceptación de unos derechos primarios, pues no podríamos considerarla aceptable independientemente de sus consecuencias, pues para Sen el argumento en contra no es la supuesta o presunta libertad que protege el mercado, sino el siguiente.

A partir de los análisis sobre las hambrunas rencia, entonces, consiste en que la cuestión no se considera como un asunto de eficiencia - económica o no-, sino como una cuestión de derechos individuales, como bien señala Damián Salcedo en su introducción citada.

Esto es, que los derechos individuales tienen —en algunas teorías - prioridad absoluta como cuestión de justicia. De modo que al evaluar las instituciones sociales, no importa lo bueno que sean los resultados que ellas produzcan, sino si tales instituciones son el resultado del ejercicio de los legítimos derechos de la persona. Así, en consecuencia, el mercado es parte del ejercicio de esos derechos y su valor depende de los propios derechos. El característico pensamiento liberal, por otra parte, sostiene que los mercados son instituciones mejores que las del Estado, pues protegen mejor los intereses de las personas al concederles la "libertad de elegir". Sin embargo, esto continúa siendo la evaluación del mercado por sus resultados, al contrario de quienes emplean un racionamiento no consecuencialista.

En síntesis, mientras que con algunos (los liberales) se puede discutir sobre si los mercados realmente arrojan las consecuencias que ellos supo- se ha analizado que muchas personas han muerto, según muestran varias experiencias, sin que hayan ocurrido bajas en la producción de alimentos a causa de desastres naturales. Más bien han ocurrido cambios bruscos en su situación (entillements) dentro del ejercicio de sus derechos legítimos, que primero llevaban a la pérdida del empleo, luego a la de la renta y luego al hambre. Comenta Sen que los desastres de este tipo se producen sin que se violen los derechos de nadie.

La pregunta inevitable para Damián Salcedo, si estos resultado ocurren, es: “¿seguirá siendo el funcionamiento del mercado aceptable, simplemente porque es parte del ejercicio legítimo de los derechos que tienen las personas? Y si no lo es, ¿por qué tendríamos que aceptar que los derechos de propiedad han de ser una consideración moral prioritaria a otras consideraciones, como las del sufrimiento o la muerte de millones de personas? Como el de cualquier otra institución social, parece que también el valor del mercado depende de sus consecuencias".

El señalamiento significativo de Sen consiste en precisar "¿de qué clase de consecuencias?". Obviamente que del pensamiento del Nobel 1998 
debemos concluir que no puede aceptar que las consecuencias se identifiquen de modo utilitarista, pues lo que él propone es que consideremos las consecuencias que las instituciones sociales tienen para la libertad.

Los tres ámbitos de la evaluación de las consecuencias de las instituciones respecto a la libertad de las personas son referidas a: (1) la oportunidad que tienen las personas para conseguir las cosas que valoran; (2) la función que tienen las personas en los procesos de toma de decisión, y (3) la inmunidad que tienen las personas frente a las posibles interferencias de otras. Es decir, como textualmente dice Sen cuando estudia "en particular tres facetas diferentes de la libertad: (a) oportunidad para conseguir algo, $(b)$ autonomía de las decisiones, o $(c)$ inmunidad frente a las intrusiones".

En la parte final de la Conferencia de John Hicks, Sen analiza la función del mecanismo de mercado en el contexto de cada una, en la sección 8 titulada "Observaciones para concluir", de la versión revisada de su conferencia, en la siguiente forma: “... de las tres facetas mencionadas resumiendo su estudio así: ... he tratado de reformular el problema de la valoración del mecanismo de mercado competitivo en términos de sus logros en el fomento de las libertades individuales, de modo opuesto a la estructura tradicional de la evaluación en términos de bienestar".

En esa reformulación, el profesor Sen concluye con el planteamiento de una interrogante fundamental en los términos siguientes: "Finalmente, ¿se ha ganado algo al pasar de la interpretación de bienestar de la eficiencia del mercado a la interpretación basada en la libertad?" y contesta categóricamente así:

Creo que hay cuatro ganancias suslanciales. En primer lugar, hay una gran distancia entre la retórica de la libertad que se usa a menudo en la literatura en defensa del mecanismo de mercado (por ejemplo, que hace a las personas 'libres para elegir' y el tratamiento exclusivamente 'bienestarista' del mecanismo de mer- cado en la economía del bienestar convencional. Es importante examinar los sentidos particulares en que $-y$ en la medida en que- el análisis económico puede sostener o no esa retórica.

En segundo lugar, la idea de libertad implica varios asuntos diferentes, entre los que se incluyen tanto los procesos y procedimientos como las oportunidades reales que las personas tienen para vivir del modo en que elegirían. Hay que distinguir entre las diferentes facetas de la libertad para alcanzar un entendimiento mejor de los diferentes modos en que es posible juzgar el fomento de la libertad. Aunque este ejercicio fue emprendido en este artículo como un preludio a un examen de lo que es posible esperar que los mercados hagan, el ejercicio tiene en sí mismo un interés más general.

En tercer lugar, en un nivel más sustantivo, se revela que los análisis de la eficiencia del mercado basados en la libertad hacen redundante el suponer que las preferencias $y$ elecciones individuales han de ser consideradas como si tuvieran por objetivo exclusivo el propio bienestar — la búsqueda del respectivo interés. Ese supuesto clásico en la evaluaciones bienestaristas se vuelve esencialmente irrelevante no sólo para la faceta de proceso de la libertad, sino también para los resultados de eficiencia en términos de libertad como oportunidad. La libertad de una persona para conseguir lo que ella prefiere (no importa por qué) nos lleva un poco más allá de este supuesto limitado y, creo, bastante erróneo.

Finalmente, al abandonar el centrarse exclusivamente en los logros de bienestar y prestar atención a los de la libertad de conseguir en general, la concepción basada en la liberetad puede animar a un cambio en la perspectiva del análisis económico técnico en una dirección que tiene considerable importancia ética y política. La relación entre mercados y libertades fue considerada por los economistas clásicos (como John Hicks notó) como un pro- 
blema trascendental y hay buenas razones en contra de ignorar completamente esa conexión. Este artículo ha prentendido clarificar algunos de los asuntos básicos de esa relación ${ }^{14}$.

En los párrafos anteriores se concretan los planteamientos fundamentales del último ganador del premio Nobel de Economía, que justifican las palabras siguientes de Johan Van Gompel a quien citamos antes: "Sen (1988) ha hecho contribuciones amplias a la 'teoría de la preferencia social', que analiza la relación entre las preferencias individuales y las decisiones colectivas. Por medio de la combinación de herramientas de la microeconomía y la filosofía, ha restaurado una dimensión ética a la economía" (traducción hecha por el autor).

Tal vez con la designación de Amartya Sen conoceremos la respuesta a la interrogante que, en la última Challenge que citamos, sirve de título a los tres trabajos sobre los que trata el premio Nobel de economía, incluidos en ese número, cuando se plantea la pregunta: “ ¿El Nobel ayuda o daña?" [Does the Nobel help or hurt?].

Belmont, California, 7 de julio de 1999.

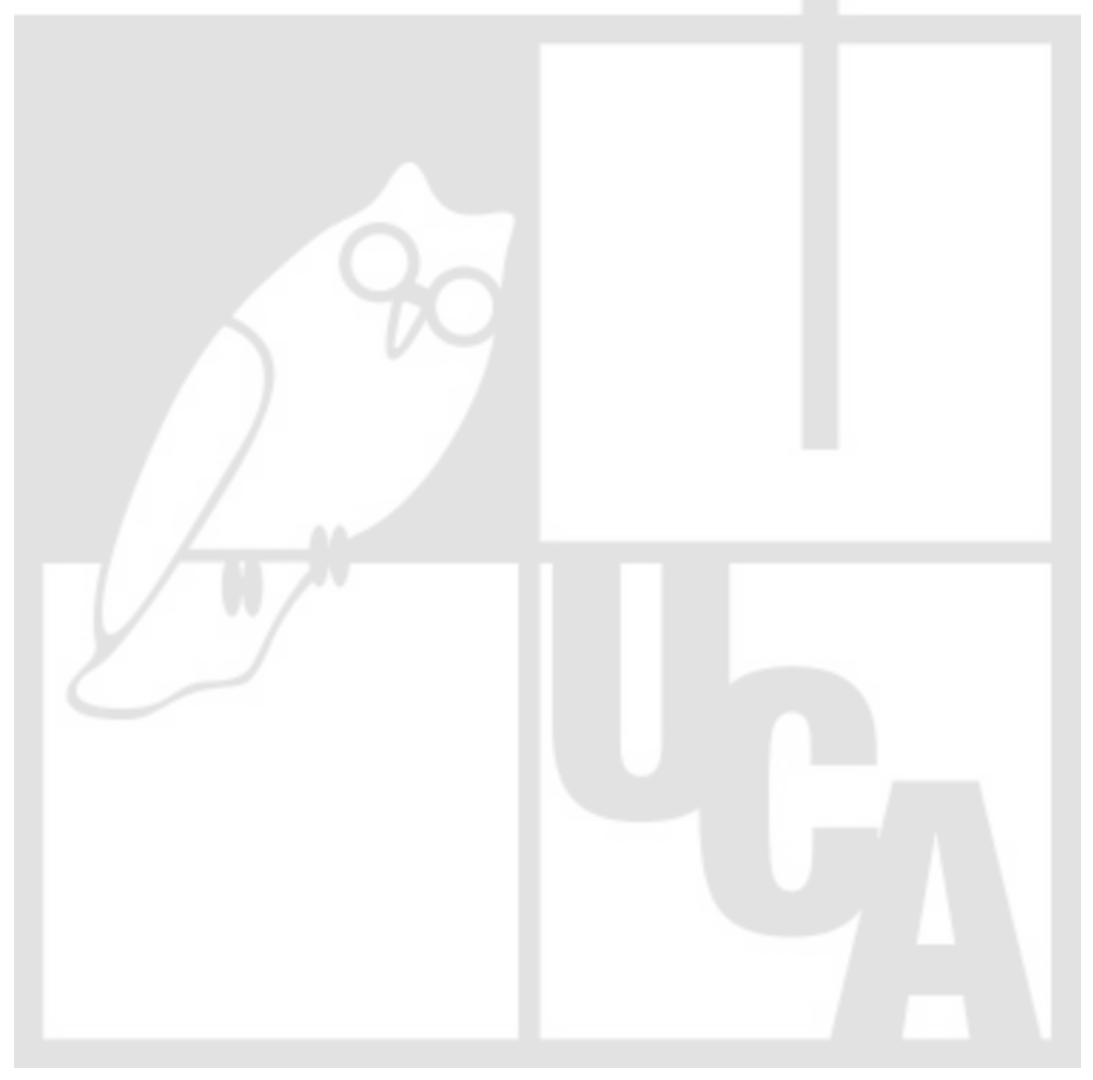

14. Amartya Kumar Sen, "Mercados y libertades. Logros y limitaciones del mecanismo de mercado en el fomento de las libertades individuales", Bienestar, justicia y mercado, pp. 151 y 152. 